This is the accepted version of the following article: Rusconi, P., Sacchi, S., Toscano, A., \& Cherubini, P. (2012). Confirming expectations in asymmetric and symmetric social hypothesis testing. Experimental Psychology, 59(5), 243-250. doi:10.1027/1618-3169/a000149, @ 2012 by Hogrefe Publishing.

This article may not exactly replicate the final version published in Experimental Psychology. It is not the version of record and is therefore not suitable for citation.

This article has been published in final form at:

http://www.psycontent.com/content/045852q385w33t67/?p=cf23f630346e4f60b0051 d7b91ca427b\&pi $=0$ 
Running Head: CONFIRMING EXPECTATIONS IN SOCIAL HYPOTHESIS TESTING

Confirming expectations in asymmetric and symmetric social hypothesis testing

Patrice Rusconi, Simona Sacchi, Armando Toscano, Paolo Cherubini Department of Psychology, University of Milano-Bicocca, Italy

Word count (text and notes): 5130 .

Author's note:

This research was supported by a grant from the Italian Department of Education, University and Research (MIUR) to Paolo Cherubini (PRIN 2008).

Correspondence concerning this article should be addressed to Patrice Rusconi, Department of Psychology, University of Milano-Bicocca, P.zza dell'Ateneo Nuovo 1, 20126, Milano, Italy. Phone ++39-02-64483736. Fax: ++39-02-64483706. E-mail:

patrice.rusconi1@unimib.it. 


\begin{abstract}
This article examines individuals' expectations in a social hypothesis testing task. Participants selected questions from a list to investigate the presence of personality traits in a target individual. They also identified the responses that they expected to receive and the likelihood of the expected responses. The results of two studies indicated that when people asked questions inquiring about the hypothesized traits that did not entail strong a priori beliefs, they expected to find evidence confirming the hypothesis under investigation. These confirming expectations were more pronounced for symmetric questions, in which the diagnosticity and frequency of the expected evidence did not conflict. When the search for information was asymmetric, confirming expectations were diminished, likely as a consequence of either the rareness or low diagnosticity of the hypothesis-confirming outcome. We also discuss the implications of these findings for confirmation bias.
\end{abstract}

Keywords: social hypothesis testing; expectations; asymmetric search; symmetric search; diagnosticity; trade-off; confirmation bias. 


\section{Confirming expectations in asymmetric and symmetric social hypothesis testing}

In everyday situations, people are called upon to recall and obtain information. Gathering incomplete or one-sided evidence may contribute to inefficiencies and errors in judgment and ultimately in the chosen course of action. Moreover, the extent and type of information that individuals search for might bias judgment even when the obtained evidence has been efficiently processed (e.g., Cameron \& Trope, 2004; Trope \& Thompson, 1997).

Hypothesis-testing tasks have been used to investigate how people obtain, test, and evaluate information (e.g., Evett, Devine, Hirt, \& Price, 1994; McKenzie, 2004; Nelson, 2005; Poletiek, 2001; Wason, 1960). In testing phases, it has been found that people tend to look for information consistent with the hypothesis rather than considering both disconfirming and confirming evidence (e.g., Baron, Beattie, \& Hershey, 1988; Cherubini, Rusconi, Russo, Di Bari, \& Sacchi, 2010; Devine, Hirt, \& Gehrke, 1990; Einhorn \& Hogarth, 1978; Evett et al., 1994; Klayman, 1995; Klayman \& Ha, 1987; Snyder \& Swann, 1978; Wason, 1960, 1966, 1968). Recent experimental research in social cognition has found that when the tested hypothesis involves strong a priori beliefs (e.g., stereotypes), subjects tend to ask asymmetric questions that allow for binary answers ("yes" vs. "no") of differing informativeness and frequency (e.g., Cameron \& Trope, 2004; Sacchi, Rusconi, Russo, Bettiga, \& Cherubini, in press; Trope \& Liberman, 1996; Trope \& Thompson, 1997). A question is symmetric when the answers that confirm or disconfirm the hypothesis are equally diagnostic and likely, provided that the prior probabilities of the hypotheses are equal. For example, testing the hypothesis that an individual is extroverted by asking "Do you like parties?" anticipates a confirming answer of "yes", which is as informative and likely as a disconfirming answer of "no". An asymmetrically confirming question (e.g., testing the hypothesis that an individual is extroverted by asking “Are you always the life of the party?") 
is a query for which a confirming answer (here, "yes") is more diagnostic but less likely than a disconfirming answer of "no", given the equal prior probabilities of the hypotheses.

Conversely, an asymmetrically disconfirming question (e.g., testing the hypothesis that an individual is extroverted by asking "Do you like to stay home alone on Saturday night?”) anticipates a more diagnostic but less likely disconfirming answer (here, "yes") than the hypothesis-confirming answer ("no"), provided equal prior probabilities of the hypotheses (e.g., Brambilla, Rusconi, Sacchi, \& Cherubini, 2011, Study 2; Cameron \& Trope, 2004; Cherubini et al., 2010; Sacchi et al., in press; Trope \& Liberman, 1996; Trope \& Thompson, 1997).

Accordingly, in contrast to symmetric questions, asymmetric questions might imply a trade-off between the diagnosticity and probability of the responses. Answers with greater probability have less diagnostic value than answers with lower probability (e.g., McKenzie, 2006; Poletiek, 2001, chaps. 1 and 2; Poletiek \& Berndsen, 2000) ${ }^{1}$. Whether people are more sensitive to the diagnosticity or probability of responses to asymmetric questions is currently under investigation. The issue is relevant because the diagnosticity/frequency trade-off has implications for confirmation bias, which can be defined as unwarranted confidence in a working hypothesis (e.g., McKenzie, 2004, 2006). Skov and Sherman (1986) and Slowiaczek, Klayman, Sherman, and Skov (1992) claim that the preference for asymmetrically disconfirming questions (which they term "extreme" tests) found in their studies indicated a tendency to confirm the working hypothesis because these questions imply that the probability of finding confirming evidence is high. In contrast, Poletiek and Berndsen (2000) suggested the preference for asymmetrically confirming questions in their tasks with realistic contexts indicated a tendency to confirm the working hypothesis because participants in their studies preferred the more diagnostic confirming evidence that this type of question provides. 
Regardless of whether the relationship between information-seeking strategies and confirmation bias is based on a tendency to maximize the probability or diagnosticity of hypothesis-confirming evidence, the motivation for using particular information-seeking strategies has often been neglected. In particular, there is little evidence regarding whether people expect to receive highly diagnostic or probable answers to their asymmetric hypothesis-confirming questions.

If individuals who prefer to ask asymmetrically disconfirming questions want to maximize the probability of receiving hypothesis-confirming answers, this informationseeking strategy would reflect confirming intentions. However, if individuals want to maximize the diagnosticity of the anticipated answers, this strategy would reflect disconfirming intentions (e.g., Poletiek \& Berndsen, 2000). Similarly, choosing an asymmetrically confirming question might reflect a desire to either maximize the informative value of a confirming answer or probability of a disconfirming answer.

Evett et al. (1994) addressed the issue of the expectations underlying the informationseeking strategies but confined their analysis to positive ("hypothesis true") and negative ("alternative true") hypothesis-testing questions. A hypothesis-testing question is positive when a positive answer ("yes") supports the working hypothesis (e.g., testing the hypothesis that an individual is extroverted by asking "Do you enjoy making new acquaintances?”). Evett et al. (1994) found that $67.6 \%$ of participants selected positive questions when testing the hypothesis that an individual was introverted/extroverted; this finding is consistent with several studies indicating that people are prone to use a positive testing strategy (e.g., Dardenne \& Leyens, 1995; Devine et al., 1990; Klayman, 1995; Klayman \& Ha, 1987; Skov \& Sherman, 1986; Snyder \& Swann, 1978). Furthermore, regardless of the hypothesis tested, more than $90 \%$ of participants expected to receive hypothesis-confirming answers (i.e., "yes" answers to positive questions and "no" answers to negative questions). 
The aim of the present study was to extend this previous investigation of an individual's expectations when testing hypotheses regarding personality traits by accounting for question symmetry or asymmetry (e.g., Cameron \& Trope, 2004; Poletiek \& Berndsen, 2000; Skov \& Sherman, 1986; Trope \& Thompson, 1997). In contrast to positivity or negativity, question symmetry or asymmetry does not reflect if the expected answers match the content of the hypothesis being tested but the extent of information that the responses convey (Cherubini et al., 2010). The question properties of positivity/negativity and symmetry/asymmetry are mutually independent (Cherubini et al., 2010). The present study thus extended the research of Evett et al. (1994) on expectations for hypothesis testing using asymmetric information-seeking strategies.

Three alternative hypotheses might account for the relationship between the information-seeking preferences of participants and their expectations. First, the use of asymmetrically confirming questions might reflect the expectation of a hypothesisdisconfirming response, whereas asymmetrically disconfirming queries might be associated with the expectation of a hypothesis-confirming response. While this hypothesis supports the view that individuals seek to maximize the probability of a hypothesis-confirming response (Skov \& Sherman, 1986; Slowiaczek et al., 1992), this type of relationship has never been hypothesized, and it seems implausible for social-inference tasks (e.g., Poletiek \& Berndsen, 2000; Sacchi et al., in press). Second, consistent with claims made by Poletiek and Berndsen (2000), participants might expect highly diagnostic responses following asymmetric questions (i.e., hypothesis-confirming answers to asymmetrically confirming questions and hypothesisdisconfirming answers to asymmetrically disconfirming questions). Third, participants' confirming expectations might be greater following symmetric questions for which confirming-answer diagnosticity and frequency do not conflict when the hypotheses are equiprobable a priori compared with the confirming expectations following both 
asymmetrically confirming and asymmetrically disconfirming questions which instead entail that the confirming answer is either rare or has low diagnosticity, respectively.

\section{Study 1}

\section{Methods}

\section{Participants}

The sample consisted of 75 undergraduate students at the University of MilanoBicocca; 52 women and 23 men between the ages of 19 and 40 years $(M=22.45, S D=3.19)$ participated. All participants were Italian citizens.

\section{Materials and procedures}

Potential participants were recruited in libraries and study rooms at the University of Milano-Bicocca and asked to participate in a study investigating social information gathering. Those who agreed then completed a questionnaire. The initial set of questions obtained demographic information (i.e., gender, age, and nationality of participants). The participants were also presented with a list of questions used to investigate whether an anonymous target individual had certain personality traits. The set of personality characteristics was based on pilot results from a prior study (Sacchi et al., in press) ${ }^{2}$. For each personality trait ("creative", "festive", "rigid", "untrustworthy", "respectful”, or "dishonest"), the list of questions consisted of three asymmetrically confirming questions (e.g., "Has she/he ever been in prison?" to test the hypothesis that the target person was dishonest), three symmetric questions (e.g., "Does she/he keep her/his word?" to test the hypothesis that the individual was untrustworthy), and three asymmetrically disconfirming questions (e.g., "Does she/he like repetitive jobs?" to test the hypothesis that the individual was creative). The symmetric and asymmetrically confirming questions were positive (i.e., a "yes" answer confirmed the working hypothesis, and a "no" answer disconfirmed it), while the asymmetrically disconfirming queries were negative (i.e., a "no" answer confirmed the hypothesis, and a 
"yes" answer disconfirmed it). The degree of asymmetry of the 54 questions used in the present study had been previously established (Sacchi et al., in press) ${ }^{3}$. Each trait and the list of questions for that trait were provided on a separate page of the questionnaire, and the six traits were presented in random order.

For each trait, participants were required to (1) select three of the nine questions to determine whether the target individual had that personality trait, (2) report whether they expected to receive a "yes" or "no" response to each of the selected questions, and (3) estimate the probability (between $0 \%$ and $100 \%$ ) that they would receive the expected answer.

Once they completed the questionnaire, participants were debriefed and thanked for their participation.

\section{Results and discussion}

Participants were required to ask about different personality traits to ensure that no specific trait would influence the results. Accordingly, all the analyses collapsed over the trait factor.

We performed an ANOVA with question type (symmetric, asymmetrically confirming, or asymmetrically disconfirming) as within-participant variable to analyze the probability that participants associated with the confirming answer (a "yes" following either asymmetrically confirming or symmetric questions and a "no" following asymmetrically disconfirming questions) averaged across the selections of the participants. When participants expected a disconfirming answer, the probability of the confirming response was calculated as the complement of the probability of the disconfirming answer. The main effect of question type was significant, $F(2,144)=3.67, M S E=139.78, p=.028, \eta^{2}=.048$, due to the significantly higher probability of confirming answers following symmetric questions compared with the probability of confirming answers following asymmetrically confirming queries, $p=.005$. Conversely, there was no significant difference between the probability of 
confirming answers following symmetrically and asymmetrically disconfirming questions, $p$ $=.21$, and between the probability of confirming answers following asymmetrically confirming compared with asymmetrically disconfirming questions, $p=.19$. Three onesample $t$-tests against the expected value of .5 showed that the probability of confirming answers following symmetric questions was significantly higher than $.5, t(73)=5.75$, twotailed $p<.001, d=.67$. Similarly, the probability of a confirming "no" answer following asymmetrically disconfirming queries was significantly higher than the expected value of .5, $t(72)=3.00$, two-tailed $p=.004, d=.35$. Conversely, there was no significant difference between the probability of a confirming answer following asymmetrically confirming questions and the expected value of $.5, t(73)=.77$, two-tailed $p=.44$ (see Figure 1$)$.

Overall, these results suggest that participants were generally driven by confirming expectations. However, these expectations differed as a function of the question type that the participants selected. Indeed, symmetric questions, which do not have the diagnosticity/frequency trade-off when the prior probabilities of the hypotheses are equal, induced higher confirming expectations. When participants chose an asymmetrically disconfirming question, they more frequently anticipated a confirming answer ("no") that was less diagnostic but more frequent compared with the more diagnostic, disconfirming answer (“yes"). This result is consistent with Skov and Sherman (1986)'s and Slowiaczek, Klayman, Sherman, and Skov (1992)'s interpretation of participants' preference for this type of question, but it is at odds with Poletiek and Berndsen (2000)'s interpretation in terms of an individuals' sensitivity to the diagnostic value of answers as opposed to the probability of occurrence. However, the participants' sensitivity to the probability of the answers was not predominant. When choosing asymmetrically confirming questions, participants may have exhibited sensitivity to the greater probability of a disconfirming answer ("no"); however, 
participants did not exhibit significantly greater expectations for disconfirming answers than more diagnostic hypothesis-confirming answers ("yes").

\section{Study 2}

In Study 1, we found that the participants' expected probability of receiving confirming answers to the questions they selected was higher than the probability of receiving disconfirming answers. This tendency varied as a function of the chosen question type. In particular, we argued that this tendency was influenced by the diagnosticity/frequency tradeoff. Study 2 was devised to investigate the confirming expectations underlying the question selection in a different manner. In particular, we attempted to generalize the results of Study 1 by comparing the response expectations for each question type when the traits under investigation were positive vs. negative and when questions were phrased as in Study 1 (hereafter, direct questions) vs. their complementary versions (hereafter, inverse questions). Furthermore, in Study 2, we wanted to gauge whether and to what extent the participants' probability of confirming answers differed from baseline levels.

\section{Methods}

\section{Participants}

Eighty-three students (36 women and $47 \mathrm{men}$ ) at the University of Milano-Bicocca volunteered to participate in the study. They ranged in age from 20 to 38 years $(M=22.8, S D$ =2.92). All participants were Italian.

\section{Materials and procedures}

We introduced two independent variables: trait valence and question phrasing. We selected four of the six traits used in Study 1 that could be converted to their opposite trait without using periphrases, namely "rigid", "untrustworthy", "respectful”, and "dishonest". Trait valence was treated as a between-groups variable; therefore, one group was presented with positive traits (i.e., "flexible", "trustworthy", "respectful ", and "honest"), and another 
group received the negative counterparts (i.e., "rigid", "untrustworthy", "disrespectful”, and “dishonest"). In addition, we manipulated the phrasing of the questions between-groups. Specifically, one group received questions identical to Study 1 (direct questions), while another group received the complementary versions of those questions (inverse questions). For example, the direct question, "Does she/he become angry if contradicted?" to inquire about target's rigidity was reversed to "Does she/he never become angry if contradicted?".

The manipulation of trait valence and question phrasing entailed a re-categorization of the asymmetrically confirming queries into asymmetrically disconfirming questions and viceversa for the questionnaires that included either negative traits and inverse questions or positive traits and direct questions relative to the classifications used in Study 1. For example, the asymmetrically disconfirming question "Has she/he never betrayed the trust of people bound to her/him?" used in Study 1 to test "untrustworthy" was re-categorized as asymmetrically confirming when the questionnaire included direct queries and the trait being tested was "trustworthy". When the questionnaire included inverse questions, the phrasing was changed to "Has she/he ever betrayed the trust of people bound to her/him?", and this was considered an asymmetrically confirming question when testing "untrustworthy".

Similarly, symmetric questions were categorized as negative (i.e., a "yes" answer falsified the hypothesized trait, while a "no" answer was hypothesis-confirming) for negative traits and inverse questions as well as for positive traits and direct questions. For questionnaires with either positive traits and inverse questions or negative traits and direct questions, the symmetric questions were categorized as positive (i.e., a "yes" answer was hypothesis-confirming).

Both task requirements and procedures were identical to Study 1.

\section{Results and discussion}


To determine which question type most frequently induced expectations for a confirming answer, we calculated (summing the three selections that participants made) the ratio of the number of each question type for which a confirming answer was expected to the total number of questions of that type. This measure allowed us to determine the extent to which each participant expected a confirming response for each question type without the confound of the participant's actual preference for selecting that question type. A $3 \times 2 \times 2$ mixed-design ANOVA with within-participant variable question type (asymmetrically confirming, symmetric, and asymmetrically disconfirming), between-participant variables trait valence (positive vs. negative), and question phrasing (direct vs. inverse) was performed. The main effect of question type was significant, $F(1,74)=5.36, M S E=.16, p=.023, \eta^{2}=$ .049 , lower-bound correction. This effect indicates that participants more frequently expected the confirming answer when choosing symmetric questions $(M=.71, S D E=.03)$ compared with either asymmetrically confirming $(M=.59, S D E=.04), p=.005$ or asymmetrically disconfirming questions $(M=.58, S D E=.04), p=.001$, while there was no difference between the expectation of confirming answers after asymmetrically confirming vs. asymmetrically disconfirming questions, $p=.81$. Furthermore, three one-sample $t$-tests against the expected value of .5 revealed that the confirming-response expectations following symmetric questions were significantly higher than $.5, t(78)=4.94$, two-tailed $p<.001, d=$ .54 , while asymmetrically confirming questions, $t(81)=1.89$, two-tailed $p=.062, d=.21$, and asymmetrically disconfirming questions, $t(81)=1.81$, two-tailed $p=.075, d=.19$, did not reach statistical significance. These findings are parallel to those of Study 1 in that symmetric questions induced expectations for confirming responses more often than the two types of asymmetric questions. Again, this might be because confirming answers to symmetric questions, as opposed to asymmetric questions, do not suffer from the diagnosticity/frequency trade-off when the prior probabilities of the hypotheses are equal, i.e., a confirming answer to 
a symmetric query might be both moderately diagnostic and frequent. By contrast, confirming answers to asymmetric questions might be either highly diagnostic but rare or highly frequent but with low diagnostic value. This might explain the lack of difference between the two types of asymmetric questions and why participants expected far less confirming answers to these questions compared with symmetric questions.

There were no significant main effects of either trait valence or question phrasing, Fs $\leq 2.61, p s \geq .11$. Neither the question type by trait valence interaction nor the question type by question phrasing interaction were significant, $F \mathrm{~s} \leq 1.75, p \mathrm{~s} \geq .19$, lower-bound correction. There was a significant trait valence by question phrasing interaction, $F(1,74)=15.65, M S E=$ $.13, p<.001, \eta^{2}=.020$, which will not be discussed further because it is beyond the scope of the present contribution. Finally, there was a significant three-way interaction between question type, trait valence, and question phrasing, $F(1,74)=27.6, M S E=.16, p<.001, \eta^{2}=$ .250. In particular, participants expected a confirming answer following the symmetric questions more frequently for inverse $(M=.98, S D E=.05)$ than direct questions $(M=.52$, $S D E=.05), p<.001$ for positive traits, while there were no differences for either asymmetrically confirming, $p=.52$, or asymmetrically disconfirming questions, $p=.99$. By contrast, for negative traits, participants' expectations for confirming-answers following symmetric questions were significantly higher for direct $(M=1.00, S D E=.06)$ than inverse questions $(M=.34, S D E=.05), p<.001$. The same tendency also applied to asymmetrically disconfirming questions, although to a lesser extent. Expectations for confirming-answers were higher for direct $(M=.61, S D E=.08)$ than inverse questions $(M=.38, S D E=.08), p=$ .050. No significant effect for question phrasing in asymmetrically confirming questions was found, $p=.18$. This finding might be because the manipulation of both trait valence and question phrasing entailed that for some symmetric questions (for which the interaction effect was greatest), the confirming answer was "yes", while for other symmetric queries, the 
confirming answer was "no". Accordingly, participants appear to be prone to more frequently expecting a confirming answer when the confirming answer was "yes" than when the confirming answer was "no". This preference for "yes" confirming answers rather than "no" might be due to the further processing step required to interpret the confirming value of "no" as opposed to "yes".

We then examined the probability that participants associated with the expected confirming answers for each question type. We compared the participants' probability estimates with the blind judges' estimates used to measure the degree of a/symmetry of questions in the Sacchi et al.'s study (in press). In particular, those judges were asked to infer the presence/absence of the behaviors described in the questions formulated by independent participants given the presence/absence of some personality traits. Accordingly, the blind judges' estimates provided us with baseline data to assess the presence and extent of the adjustments that participants' expectations induced in the current study.

Specifically, for each question and trait, we computed the probability of receiving a confirming/disconfirming answer by averaging the blind judges' estimates. From a formal (Bayesian) standpoint, this required the computation of $p(D)$ for each blind judge (the probability of receiving a confirming answer to a question or in a logically equivalent phrasing, the probability of occurrence of a behavior). This was determined by the following equation:

$$
p(D)=p(D \mid H) \times p(H)+p(D \mid \neg H) \times p(\neg H),
$$

where $p(D \mid H)$ and $p(D \mid \neg H)$ are the probabilities of observing a behavior $(D)$ given that the hypothesized trait is true $(H)$ or false $(\neg H)$, respectively. Each conditional probability is weighted by the prior probability of observing the hypothesized trait in the population $(p(\neg H)$ was computed as the complement of $p(H))$. 
For negative queries, the probability associated with a confirming answer was computed as follows:

$$
p(\neg D)=1-p(D)
$$

Finally, we calculated the probability $\Delta$ for a confirming answer for each participant, question, and trait combination. Specifically, $\Delta$ was computed as follows:

$\Delta=$ participants' estimate - mean blind judges' estimates.

Accordingly, when $\Delta$ assumes a value of zero, there is alignment between participants and judges. When $\Delta$ assumes positive values, participants' estimates are overestimated compared with the baseline values, while when $\Delta$ is negative, participants' estimates are underestimated compared with the baseline values. Confirming expectations are reflected by positive $\Delta$ values.

As in Study 1, the participants' probability estimates were averaged across the selections that participants made, and the estimates were recoded to ensure that when a disconfirming answer was expected, the probability of the confirming answer was computed as its complement. Using $\Delta$ as dependent variable, we performed a $3 \times 2 \times 2$ mixed-design ANOVA using question type (asymmetrically confirming, symmetric, asymmetrically disconfirming) as the within-participant variable and question valence (positive vs. negative) and question phrasing (direct vs. inverse) as between-participant variables. Only the question type demonstrated significant main effects, $F(1,76)=10.24, M S E=533.22, p=.002, \eta^{2}=$ .111 , lower-bound correction. Neither the main effect of question valence nor the main effect of question phrasing were significant, $F \mathrm{~s} \leq 2.5, p \mathrm{~s} \geq .12$. No interaction reached the significance level, $F \mathrm{~s} \leq 3.31, p \mathrm{~s} \geq .07$, lower-bound correction. The significant main effect of question type was due to the positive $\Delta$ found for symmetric questions, which was significantly higher than both the negative $\Delta \mathrm{s}$ associated to both types of asymmetric queries (i.e., either confirming or disconfirming), $p \mathrm{~s}<.001$. By contrast, no significant difference 
emerged between the $\Delta$ s associated to asymmetrically confirming vs. asymmetrically disconfirming questions, $p=.49$. Three one-sample $t$-tests against the expected value of 0 confirmed that the $\Delta$ relative to symmetric questions was significantly higher than zero, $t(82)$ $=4.27$, two-tailed $p<.001, d=.47$, while the $\Delta$ s relative to either asymmetrically confirming, $t(80)=-.91$, two-tailed $p=.37$, or asymmetrically disconfirming questions, $t(81)=-.01$, twotailed $p=.99$, did not significantly differ from zero (see Figure 2 ). Therefore, the comparison between the probability estimates of expected confirming answers (participants) vs. confirming behaviors (judges) dovetailed with the results of the previous analyses, showing that symmetric questions induced confirming expectations to a significantly greater extent than either asymmetrically confirming or asymmetrically disconfirming questions. In particular, the finding that the $\Delta \mathrm{s}$ of asymmetrically confirming and asymmetrically disconfirming questions did not significantly differ from zero (i.e., the value that indicates calibration) or each other demonstrated that both disadvantages entailed by the diagnosticity/frequency trade-off (i.e., either the rareness or low diagnosticity of the outcome) are perceived as equally important by participants. However, although not significant, the comparison between the two types of asymmetric queries again revealed that participants tended to favor the more likely rather than the more diagnostic outcome (see Figure 2).

\section{Discussion}

This study investigated if the use of asymmetric or symmetric hypothesis-testing strategies when making social inferences was based on the expectation of receiving a hypothesis-confirming answer. In recent studies of social cognition, it has been argued that the use of an asymmetrically confirming strategy (i.e., asking questions for which the confirming answer is more diagnostic than the disconfirming answer) leads to a bias in favor of the hypothesis being tested because a disconfirming answer only weakly falsifies the working hypothesis compared with the strong support provided by a confirming answer (e.g., 
Trope \& Thompson, 1997). However, an asymmetric search strategy implies a trade-off between the diagnosticity and probability of the anticipated answers when the prior probabilities of the hypotheses are equal (e.g., McKenzie, 2006; Poletiek, 2001, chaps. 1 and 2; Poletiek \& Berndsen, 2000). For asymmetrically confirming questions, confirming answers, while more diagnostic than the disconfirming answers, are also more rare. For example, a hypothesis-confirming answer ("yes") to the question "Have you ever been in prison?" is more diagnostic when testing the hypothesis that an individual is dishonest than the hypothesis-disconfirming answer ("no") because one might be dishonest without having been in prison. However, the hypothesis-confirming answer ("yes") is also less common than a "no" response because fewer people have been in prison compared with those who have never been in prison. Whether people are more sensitive to the diagnosticity or probability of the anticipated answers is currently being debated (e.g., Poletiek \& Berndsen, 2000; Skov \& Sherman, 1986) and is the focus of recent experimental research (Sacchi et al., submitted). Identifying which answers people expect to receive to their hypothesis-testing questions is crucial for determining the relationship between information-seeking strategies and confirmation bias.

While Evett et al. (1994) addressed this issue, their analysis focused only on the use of positive or negative questions, which do not have the same implications for confirmation bias as asymmetric queries. Positive questions do not necessarily entail confirming tendencies, although they might reveal inefficiencies in information gathering (e.g., Klayman, 1995; Klayman \& Ha, 1987; McKenzie, 2004; Trope \& Thompson, 1997). Furthermore, positivity/negativity and symmetry/asymmetry are independent properties of questions (Cherubini et al., 2010).

The present study was designed to investigate the expectations underlying the selection of asymmetric or symmetric questions. To judge whether a target person possessed a 
particular personality trait, participants were required to select questions from a list composed of symmetric, asymmetrically confirming, and asymmetrically disconfirming questions and to report the expected answer and its likelihood to the selected questions. Overall, participants had greater confirming-answer expectations following the selection of symmetric questions compared with choosing both types of asymmetric queries. This result also emerged when the valence of the traits under investigation and the phrasing of the questions (which were also presented to participants with a inverse phrasing) were manipulated. We argue that the higher confirming expectations following symmetric questions might be due to the absence of the diagnosticity/frequency trade-off because symmetric questions entail moderate diagnostic and frequent confirming answers when the prior probabilities of the hypotheses are equal.

The lack of difference between asymmetrically confirming and asymmetrically disconfirming questions in inducing expectations for confirming answers was also consistent between both studies. We interpreted this finding in terms of participants' perception of both types of disadvantages (i.e., rareness and low diagnosticity) entailed by the diagnosticity/frequency trade-off. It appears as though participants treated the expectations of a rare confirming response and those of a low diagnostic confirming response equally. Participants exhibited a tendency to be more sensitive to the probability of outcome occurrence, shown by the higher probabilities associated with confirming answers following asymmetrically disconfirming vs. asymmetrically confirming queries in Study 1 and the comparison against the expected value of zero, which was significant for the (likely) confirming responses to asymmetrically disconfirming questions, but not for the (highly diagnostic) confirming responses to asymmetrically confirming questions (see Figure 1).

Future research should explore whether the confirming expectations found in this social information-gathering task have implications for evaluating the answers provided by a target individual and overall judgment and impression formation. It has been argued that a 
confirmation bias can only be determined if people exhibit particular combinations of information-seeking bias and bias in evidence evaluation (e.g., Klayman, 1995; McKenzie, 2004, 2006). The implications of confirming expectations for intergroup processes should also be investigated. This issue is especially relevant for understanding the psychological mechanisms underlying the resistance to stereotype change (e.g., Johnston, 1996; Johnston, Hewstone, Pendry, \& Frankish, 1994; Moreno \& Bodenhausen, 1999). 


\section{References}

Baron, J., Beattie, J., \& Hershey, J. C. (1988). Heuristics and biases in diagnostic reasoning: II. Congruence, information, and certainty. Organizational Behavior and Human Decision Processes,42, 88-110. doi:10.1016/0749-5978(88)90021-0

Brambilla, M., Rusconi, P., Sacchi, S., \& Cherubini, P. (2011). Looking for Honesty: The Primary Role of Morality (vs. Sociability and Competence) in Information Gathering. European Journal of Social Psychology, 41, 135-143. doi: 10.1002/ejsp.744.

Cameron, J. A., \& Trope, Y. (2004). Stereotype-biased search and processing of information about group members. Social Cognition, 22, 650-672. doi:

$10.1521 /$ soco.22.6.650.54818

Cherubini, P., Rusconi, P., Russo, S., Di Bari, S., \& Sacchi, S. (2010). Preferences for different questions when testing hypotheses in an abstract task: Positivity does play a role, asymmetry does not. Acta Psychologica, 134, 162-174.

doi:10.1016/j.actpsy.2010.01.007

Dardenne, B., \& Leyens, J.-Ph. (1995). Confirmation bias as a social skill. Personality and Social Psychology Bulletin, 21, 1229-1239. doi:10.1177/01461672952111011

Devine, P. G., Hirt, E. R., \& Gehrke, E. M. (1990). Diagnostic and confirmation strategies in trait hypothesis testing. Journal of Personality and Social Psychology, 58, 952-963. doi: $10.1037 / 0022-3514.58 .6 .952$

Einhorn, H. J., \& Hogarth, R. M. (1978). Confidence in judgment: Persistence of the illusion of validity. Psychological Review, 85, 395-416. doi:10.1037//0033-295X.85.5.395

Evett, S. R., Devine, P. G., Hirt, E. R., \& Price, J. (1994). The role of the hypothesis and the evidence in the trait hypothesis testing process. Journal of Experimental Social Psychology, 30, 456-481. doi: 10.1006/jesp.1994.1022 
Johnston, L. (1996). Resisting change: Information seeking and stereotype change. European Journal of Social Psychology, 26, 799-826. doi:10.1002/(SICI)10990992(199609)26:5<799::AID-EJSP796>3.3.CO;2-F

Johnston, L., Hewstone, M., Pendry, L., \& Frankish, C. (1994). Cognitive models of stereotype change (4): Motivational and cognitive influences. European Journal of Social Psychology, 24, 237-266. doi: 10.1002/ejsp.2420240203

Klayman, J. (1995). Varieties of confirmation bias. The Psychology of Learning and Motivation, 32, 385-418. doi: 10.1016/S0079-7421(08)60315-1

Klayman, J., \& Ha, Y. W. (1987). Confirmation, disconfirmation, and information in hypothesis testing. Psychological Review, 94, 211-228. doi: 10.1037/0033295X.94.2.211

McKenzie, C. R. M. (2004). Hypothesis testing and evaluation. In D. J. Koehler \& N. Harvey (Eds.), Blackwell handbook of judgment and decision making (pp. 200-219). Malden, MA, US: Blackwell Publishing.

McKenzie, C. R. M. (2006). Increased sensitivity to differentially diagnostic answers using familiar materials: Implications for confirmation bias. Memory \& Cognition, 34, $577-$ 588. doi:10.3758/BF03193581

Moreno, K. N., \& Bodenhausen, G. V. (1999). Resisting stereotype change: The role of motivation and attentional capacity in defending social beliefs. Group Processes and Intergroup relations, 2, 5-16. doi: 10.1177/1368430299021001

Poletiek, F. [H.] (2001). Hypothesis-testing behaviour. Hove, U.K.: Psychology Press.

Poletiek, F. H., \& Berndsen, M. (2000). Hypothesis Testing as Risk Behaviour with Regard to Beliefs. Journal of Behavioral Decision Making, 13, 107-123. doi: 10.1002/(SICI)1099-0771(200001/03)13:1<107::AID-BDM349>3.0.CO;2-P 
Sacchi, S., Bonomi, M., Rusconi, P., \& Cherubini, P. (submitted). Asymmetric information gathering on social targets: A trade-off between evidence diagnosticity and frequency.

Sacchi, S., Rusconi, P., Russo, S., Bettiga, R., \& Cherubini, P. (in press). New knowledge for old credences: Asymmetric information search about in-group and out-group members. British Journal of Social Psychology. doi:10.1111/j.20448309.2011.02026.x

Skov, R. B., \& Sherman, S. J. (1986). Information-gathering processes: Diagnosticity, hypothesis-confirmatory strategies, and perceived hypothesis confirmation. Journal of Experimental Social Psychology, 22, 93-121. doi: 10.1016/0022-1031(86)90031-4

Slowiaczek, L. M., Klayman, J., Sherman, S. J., \& Skov, R. B. (1992). Information selection and use in hypothesis testing: What is a good question, and what is a good answer? Memory \& Cognition, 20, 392-405. doi:10.3758/BF03210923

Snyder, M., \& Swann, W. B. (1978). Hypothesis-testing processes in social interaction. Journal of Personality and Social Psychology, 36, 1202-1212. doi:10.1037/00223514.36.11.1202

Trope, Y., \& Liberman, A. (1996). Social hypothesis-testing: Cognitive and motivational mechanisms. In E. T. Higgins \& A. W. Kruglanski (Eds.), Social psychology: Handbook of basic principles (pp. 239-270). New York: Guilford Press.

Trope, Y., \& Thompson, E. P. (1997). Looking for truth in all the wrong places? Asymmetric search of individuating information about stereotyped group members. Journal of Personality and Social Psychology, 73, 229-241. doi: 10.1037/0022-3514.73.2.229

Wason, P. C. (1960). On the failure to eliminate hypotheses in a conceptual task. The Quarterly Journal of Experimental Psychology, 12, 129-140. doi:10.1080/17470216008416717 
Wason, P. C. (1966). Reasoning. In B. M. Foss (Ed.), New horizons in psychology I.

Harmondsworth, UK: Penguin.

Wason, P. C. (1968). Reasoning about a rule. The Quarterly Journal of Experimental Psychology, 20, 273-281. doi: 10.1080/14640746808400161 


\section{Footnotes}

${ }^{1}$ Note that the diagnosticity/frequency trade-off cannot be generalized to all contexts in which people formulate questions. In particular, when the prior probabilities of the hypotheses are unequal (for example, when people rely on stereotype-based knowledge; see, e.g., Trope \& Thompson, 1997), there might not be a trade-off between diagnosticity and frequency (e.g., a hypothesis-confirming answer to an asymmetrically confirming query might be both highly diagnostic and frequent). Therefore, our analysis is confined to cases in which no strong a priori beliefs are involved. Participants in the present study were asked to estimate the a priori probability of a social target to possess the hypothesized traits. Their mean rating on 1 to 7 scales fluctuated around the midpoint (i.e., 4.05 in Study 1 and 3.65 in Study 2), indicating that they were not influenced by strong a priori beliefs in their judgments.

${ }^{2}$ We used the six traits pretested in Sacchi et al. (in press), specifically "rigid", "respectful", "festive", "untrustworthy", "dishonest", and "creative" because they were balanced in terms of valence, and the asymmetry indexes of the questions presented to participants in the present experiment were calibrated relative to these traits.

${ }^{3}$ Sacchi et al. (in press) computed the questions' asymmetry indexes based on the estimates provided by the 74 judges asked to make inferences with regards to both the questions produced by the 37 independent participants and their corresponding answers. The judges were unaware of the target of the questions generated by the other participants. The judges' estimates were used to compute the asymmetry index according to the following formula (the same formula was used by Trope \& Thompson, 1997): $p(H \mid D)-p(\neg H \mid \neg D)$, where $p(H \mid D)$ is the conditional probability of possessing the hypothesized trait given a hypothesis-confirming answer to the question and $p(\neg H \mid \neg D)$ is the conditional probability of not having the trait given a hypothesis-disconfirming answer to the same question. For 
pragmatic reasons (i.e., to avoid the use of a double negative), $p(\neg H \mid \neg D)$ was computed as the complement of $p(H \mid \neg D)$. Questions in which the asymmetry index was closest to 0 were chosen as symmetric questions, those with an asymmetry index closest to 1 were selected as asymmetrically confirming questions, and those with an asymmetry index closest to -1 were chosen as asymmetrically disconfirming queries. The asymmetry polarization of the questions was balanced. 


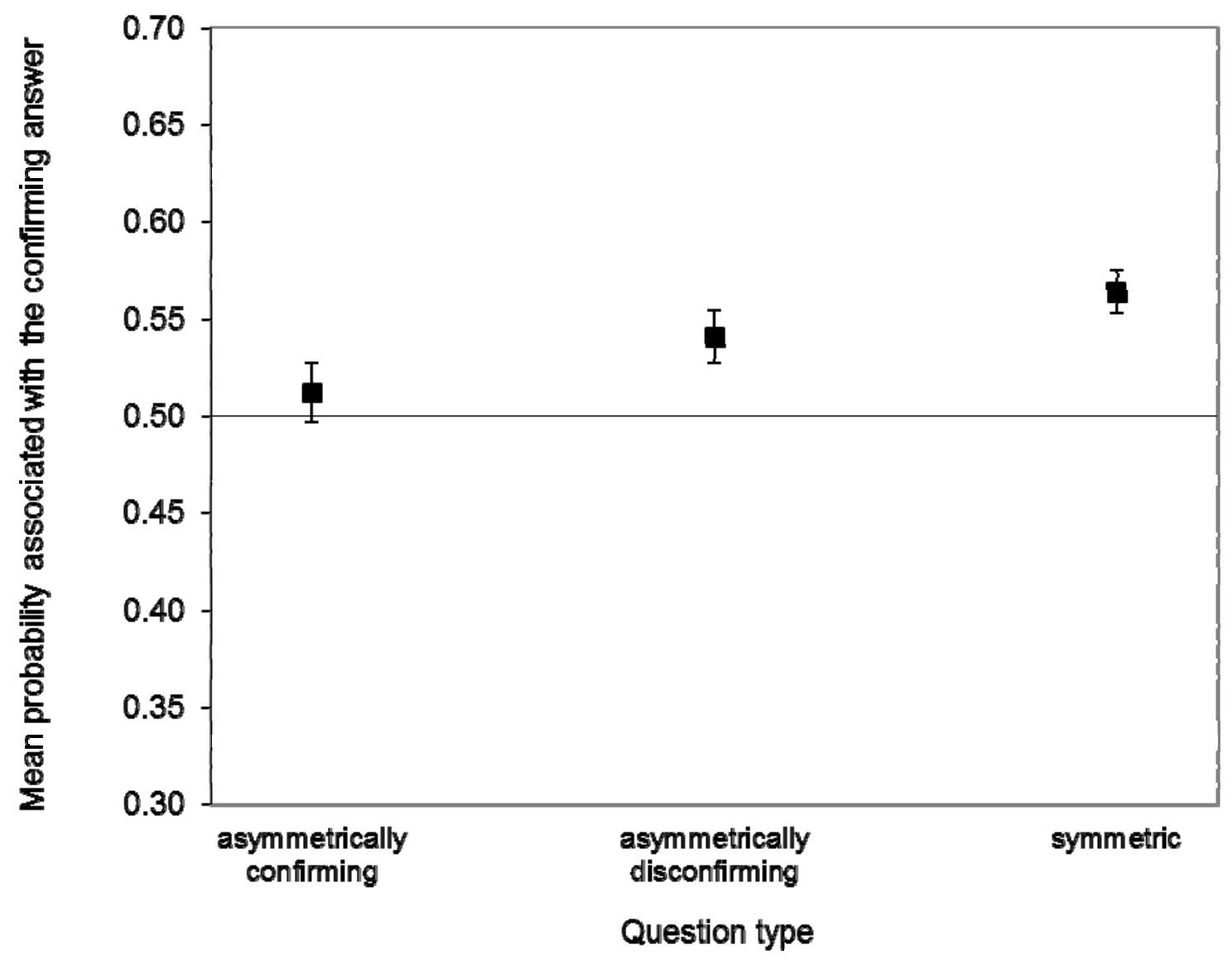

Figure 1. Mean probability associated with the confirming answer for each question type in Study 1. Error bars represent the standard errors of the means (SEMs). 


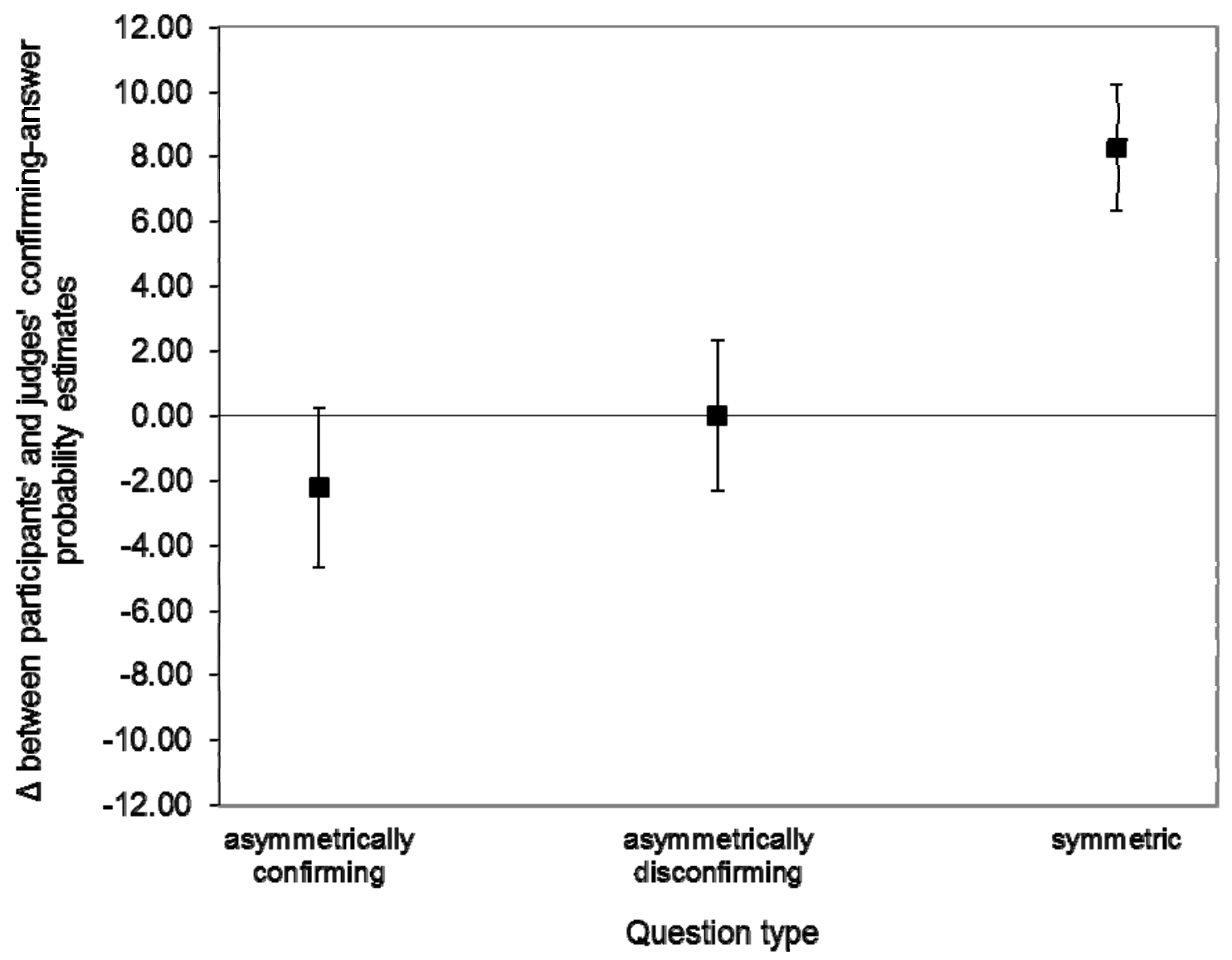

Figure 2. The $\Delta$ between the participants' and judges' confirming-answer probability estimates for each question type in Study 2. Error bars represent the standard errors of the means (SEMs). 